\title{
TTF1-NP induces protective autophagy during apoptosis by inhibiting the Akt/mTOR pathway and activating JNK in human liver cancer cells
}

\author{
XUAN ZHANG $^{1 *}$, MEILAN ZHANG $^{2 *}$, BIN XIAO $^{1}$, WENJUN JIAO ${ }^{1}$, SILIN ZHANG $^{1}$ and XUEWU ZHANG ${ }^{1}$ \\ ${ }^{1}$ College of Medicine, Yanbian University; ${ }^{2}$ The Affiliated Hospital of Yanbian University, Yanji, Jilin, P.R. China
}

Received July 30, 2017; Accepted January 2, 2018

DOI: $10.3892 /$ or.2018.6196

\begin{abstract}
TTF1-NP is a flavonoid nanoparticle based on 5,2',4'-trihydroxy-6,7,5'-trimethoxyflavone (TTF1), which is derived from the medicinal plant Sorbaria sorbifolia that grows in the Changbai Mountain. We previously demonstrated antitumor effects of TTF1-NP in human hepatoma including induction of apoptosis and inhibition of angiogenesis, migration and invasion. Herein, we examined the effects of TTF1-NP on autophagy and its relationship with apoptosis, and explored potential underlying mechanisms in human hepatoma cell lines. We conducted cell viability assays, Annexin V/propidium iodide double staining, Hoechst staining, monodansylcadaverine staining, transmission electron microscopy, green fluorescent protein-light chain 3 plasmid transfection and western blots. We found that TTF1-NP induced apoptosis and autophagy in HepG2 and SMMC-7721 cells. Pretreatment with the autophagy inhibitor 3-methyladenine promoted TTF1-NP-induced apoptosis. TTF1-NP decreased levels of phosphorylated (p)-Akt, p-mTOR and p-ERK1/2 and increased p-JNK levels in the two cell lines. Treating cells with insulin, SP600125 and U0126 indicated that the Akt/mTOR pathway and JNK were involved in TTF1-NP-induced autophagy. Together, these findings suggest that TTF1-NP induced protective apoptosis-related autophagy by modulating the Akt/mTOR and JNK pathways in HepG2 and SMMC-7721 cells. Therefore, autophagy may be a potential target for TTF1-NP in human hepatoma therapy.
\end{abstract}

\section{Introduction}

TTF1 (5,2',4'-trihydroxy-6,7,5'-trimethoxyflavone) is a flavonoid compound derived from Sorbaria sorbifolia, which

Correspondence to: Professor Xuewu Zhang, College of Medicine, Yanbian University, No. 977 Gongyuan Road, Yanji, Jilin, P.R. China E-mail: zhangxuewu@ybu.edu.cn

${ }^{*}$ Contributed equally

Key words: Sorbaria sorbifolia, TTF1-NP, apoptosis, autophagy, Akt/mTOR, MAPK, hepatoma grows in the Changbai Mountains and was used as a medicinal plant to treat swelling and inflammation in ancient China (1). TTF1 is the major bioactive anticancer compound in $S$. sorbifolia: It has been shown to inhibit angiogenesis induced by HepG2 cells in chick embryo chorioallantoic membranes and to induce apoptosis through a mitochondrial pathway in HepG2 cells $(2,3)$. However, the use of TTF1 as an anticancer drug has been limited by its high biodegradability. TTF1 nanoparticles (TTF1-NP) are prepared by an emulsion evaporation-solidification method at a low temperature and are more soluble and susceptible to absorption (1). We previously demonstrated that TTF1-NP has an anti-hepatoma effect associated with induction of apoptosis and inhibition of angiogenesis, migration and invasion in human hepatoma cells and tissues $(4,5)$.

Similar to apoptosis, autophagy plays an important role in the regulation of cancer development and may be a target for cancer therapies. Autophagy is the process by which eukaryotic cells degrade and recycle misfolded proteins and impaired organelles (6). It begins with a semi-closed isolation membrane that subsequently develops into a phagosome. In response to drugs or environmental stimuli, the phagosome is converted into an autophagosome with a double-membrane that contains broken organelles or cytosol. Autophagosomes then fuse with lysosomes and their contents are degraded (7). In recent years, many efforts have been made to identify autophagy-related genes with the aim of elucidating the mechanism of autophagosome formation. Several autophagy-related genes have been identified in yeast and orthologs have been found in higher eukaryotes. However, the relationship between apoptosis and autophagy in tumors is complex. Various studies have implicated that autophagy is involved in the control of both cell survival and death in different cell types or in response to diverse drug treatments (8-10).

The apoptosis and autophagy responses to diverse stressors share many pathways. For example, the protein kinase $\mathrm{B} / \mathrm{mammalian}$ target of rapamycin (Akt/mTOR) pathway and the mitogen-activated protein kinase (MAPK) pathway that includes extracellular signal-regulated kinase (ERK), p38 MAPK and c-Jun-N-terminal kinase (JNK) play vital roles not only in apoptosis, but also in autophagy in cancer cells $(11,12)$. Several studies have shown that the Akt/mTOR and MAPK pathways are essential to regulation of autophagy in liver cancer cells $(13,14)$. However, studies of TTF1-NP-induced autophagy 
and the interplay between autophagy and apoptosis in human hepatoma are scarce. Thus, we studied the effect of TTF1-NP on autophagy and the relationship between autophagy and apoptosis, and examined potential mechanisms, focusing on the $\mathrm{Akt} / \mathrm{mTOR}$ and MAPK pathways in liver cancer cells.

\section{Materials and methods}

Preparation of TTF1-NP. TTF1-NP (Fig. 1A) was prepared based on previous studies (1-3). TTF1 (380 mg) was obtained from $10 \mathrm{~kg}$ of Sorbaria sorbifolia (collected from Yanji, China) by using water extraction and alcohol precipitation (2). TTF1 was encapsulated in stearic acid solid lipid nanostructured carrier (average particle diameter, 195.2 $\pm 35.2 \mathrm{~nm}$; average entrapment rate, $64.57 \pm 8.21 \%$ ) in an emulsion evaporation-solidification method at low temperature (1). TTF1-NP with stearic acid solid lipid nanostructure was more soluble than TTF1 $(1,3)$.

Reagents. Dulbecco's modified Eagle's medium (DMEM), fetal bovine serum (FBS) and penicillin-streptomycin were purchased from Gibco-BRL (Grand Island, NY, USA). Rabbit-polyclonal antibodies of LC3B I/II, Beclin-1, p-Akt (Ser473), Akt, p-mTOR (Ser2448), mTOR, p-JNK (Thr183/Tyr185) were purchased from Abcam. Rabbit-polyclonal antibodies of p-p38 MAPK (Thr180/Tyr182), p-ERK1/2 (Thr202/Tyr204), active-caspase-3, p62 and $\beta$-actin were purchased from Cell Signaling Technology (Danvers, MA, USA). The chemicals used were 3-(4,5-dimethylthiazol-2-yl)-2,5-diphenyltetrazolium bromide (MTT) (Sigma, St. Louis, MO, USA), MDC (KeyGen Co. Ltd., Nanjing, China), 3-methyladenine (MedChemExpresss, Monmouth Junction, NJ, USA), Hoechst 33258 (Beyotime, Jiangsu, China), insulin (Beyotime), SP600125 (Beyotime), and U0126 (Beyotime).

Cell culture and viability assay. HepG2 and SMMC-7721 cells were purchased from KeyGen Co. Ltd. and cultured in DMEM with $10 \% \mathrm{FBS}, 100 \mathrm{U} / \mathrm{ml}$ penicillin-streptomycin at $37^{\circ} \mathrm{C}$ in a humidified $5 \% \mathrm{CO}_{2}$ incubator. Cells were plated in 96-well plates $\left(5 \times 10^{3} / 100 \mu \mathrm{l} /\right.$ well $)$ and incubated with TTF1-NP $(0,40$, $80,120 \mu \mathrm{M})$. MTT $(5.0 \mathrm{mg} / \mathrm{ml}, 20 \mu \mathrm{l})$ was added after the cells were incubated for $24 \mathrm{~h}$, then $150 \mu \mathrm{l}$ DMSO was added into each well. The optical density (OD) was measured at $490 \mathrm{~nm}$, respectively. The cell viability value was calculated using the following formula: OD sample/OD blank x $100 \%$.

Fluorescent staining. HepG2 and SMMC-7721 cells grown in 6-well plates were treated with TTF1-NP for $24 \mathrm{~h}$, incubated with $1 \mathrm{mM}$ MDC for $30 \mathrm{~min}$ or Hoechst 33258 for $20 \mathrm{~min}$ and washed with phosphate-buffered saline (PBS). Fluorescence images were captured by fluorescence microscope (Olympus, Tokyo, Japan).

Transmission electron microscopy (TEM). Cells were treated with TTF1-NP ( $80 \mu \mathrm{M})$, trypsinized and washed with PBS before fixed in fixative buffer. Subsequently, cells were collected at 1,000 r/min for $10 \mathrm{~min}$, and then incubated in $3 \%$ glutaraldehyde. The next day, the samples were treated with $2 \%$ osmium tetroxide, followed by dehydrating through a graded series of acetone, and embedded in resin. Finally, the samples were sliced into 60-nm sections and prepared for TEM (Hitachi, Ltd., Tokyo, Japan).

Annexin V/PI double staining. HepG2 and SMMC-7721 cells were seeded into a 6 -well plate $\left(3 \times 10^{5}\right.$ cells/well), and treated with the indicated amounts of TTF1-NP for $24 \mathrm{~h}$. Cells were collected, washed in cold PBS and resuspended in binding buffer at a concentration of $1 \times 10^{6}$ cells $/ \mathrm{ml}$. Then, $5 \mu \mathrm{l}$ Annexin V-FITC and $10 \mu \mathrm{l}$ PI were added in $100 \mu \mathrm{l}$ cell suspension and incubated for $15 \mathrm{~min}$. After added $400 \mu \mathrm{l} \mathrm{PBS}$, the samples were detected by flow cytometry. The results were analyzed with the BD FACSCalibur ${ }^{\mathrm{TM}}$ system.

Transfection of green fluorescent protein-light chain 3 (GFP-LC3) plasmid. The GFP-LC3 plasmid was kindly provided by Dr Quan Zhang (Yangzhou University, Yangzhou, China). According to manufacturer's protocol of Lipofectamine 2000 (Invitrogen), HepG2 and SMMC-7721 cells were transfected with GFP-LC3 plasmid. After indicated treatment of TTF1-NP, the cells with GFP-LC3 puncta were detected under fluorescence microscope (Olympus, Tokyo, Japan).

Western blot analysis. After treatment HepG2 and SMMC-7721 cells with various concentrations of TTF1-NP, the total protein was obtained by RIPA lysis buffer (Solarbio, Beijing, China). The proteins were separated by sodium dodecyl sulfate-polyacrylamide gel electrophoresis (SDS-PAGE) (100 V, $120 \mathrm{~min}$ ) and transferred to a polyvinylidene fluoride (PVDF) membrane (100 V, 30-90 min). Subsequently, the membranes were blocked with skimmed milk (5\%) and then incubated overnight at $4^{\circ} \mathrm{C}$ with the following antibodies: Akt $(1: 1,000)$, mTOR (1:1,500), p-Akt (1:5,000), p-mTOR (1:2,000), LC3B I/II (1:500), Beclin-1 (1:1,000), p62 (1:5,000), p-JNK (1:1,000), p-ERK1/2 (1:1,000), p-p38 MAPK $(1: 1,000), \beta$-actin $(1: 1,000)$ and active-caspase-3 (1:1,000). The next day, the membranes were incubated with an anti-rabbit secondary antibody $(1: 1,500)$ for $2 \mathrm{~h}$ at room temperature. After ECL incubation, the target proteins were tested using Bio-Rad imaging system (Bio-Rad, Hercules, CA, USA).

Statistical analysis. The experiments were repeated three times. The data are expressed as the mean \pm SD, and differences between groups were analyzed by one-way analysis of variance (ANOVA) and Student's t-test. The results were considered statistically significant when the P-value was $<0.05$.

\section{Results}

TTF1-NP induces apoptosis in HepG2 and SMMC-7721 cell lines. We previously reported TTF1-NP cytotoxicity in various human liver cancer cell types (5). Herein, to examine the effect of TTF1-NP on cell viability, HepG2 and SMMC-7721 were treated with 0-120 $\mu \mathrm{M}$ TTF1-NP for $24 \mathrm{~h}$, and then MTT assays were performed. TTF1-NP decreased HepG2 and SMMC-7721 cell viability in a dose-dependent manner (Fig. 1B). The $\mathrm{IC}_{50}$ values were $88.27 \mu \mathrm{M}$ for HepG2 and $90.39 \mu \mathrm{M}$ for SMMC-7721 cells at $24 \mathrm{~h}$. Hoechst staining and western blotting showed that apoptosis-related bright blue fluorescence in the nuclei of cells and levels of the apoptosis-related protein active caspase-3, respectively, increased with increasing TTF1-NP concentration 
A

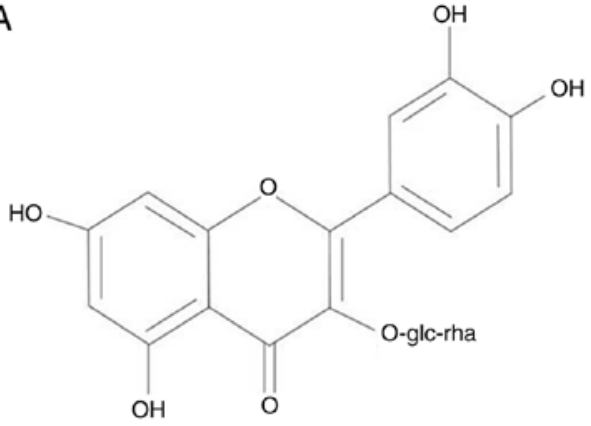

B

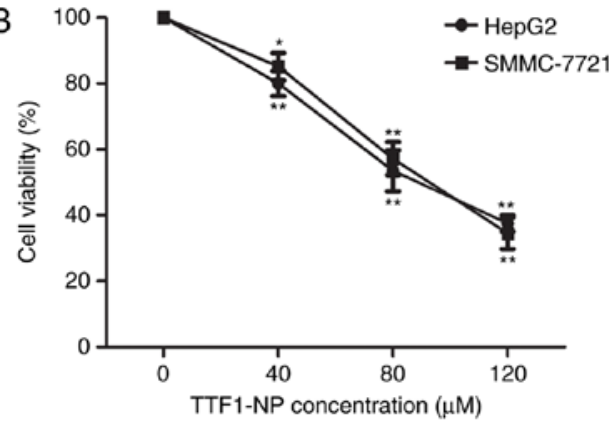

C
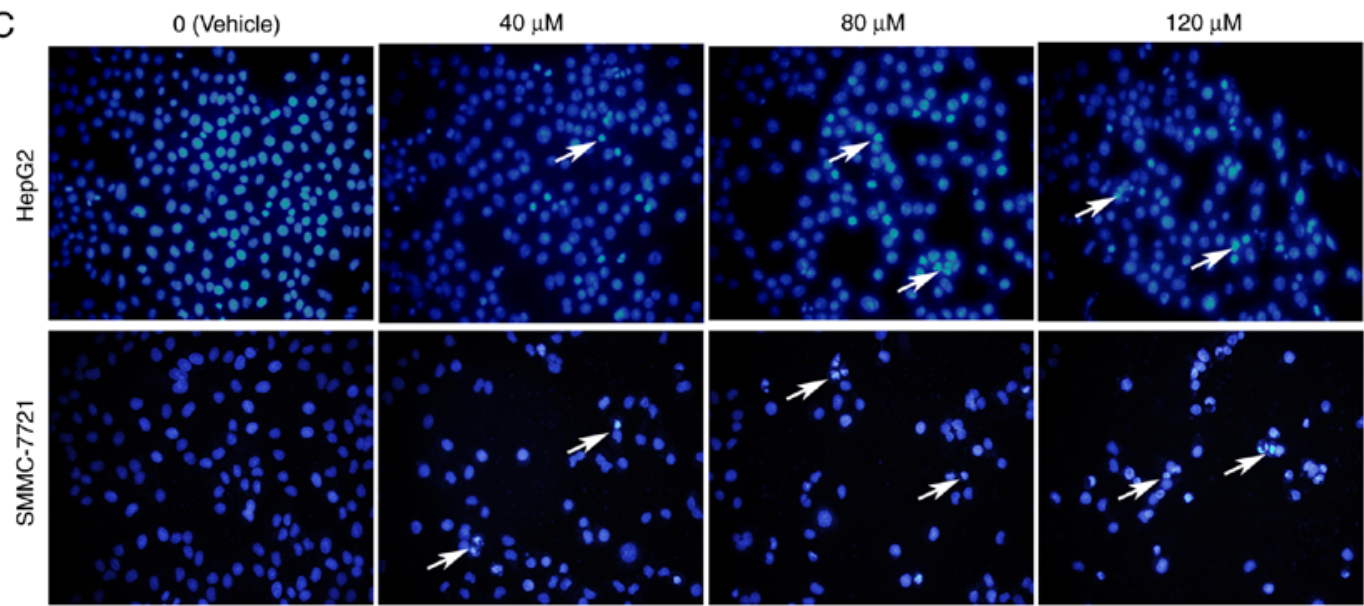

$\mathrm{D}$
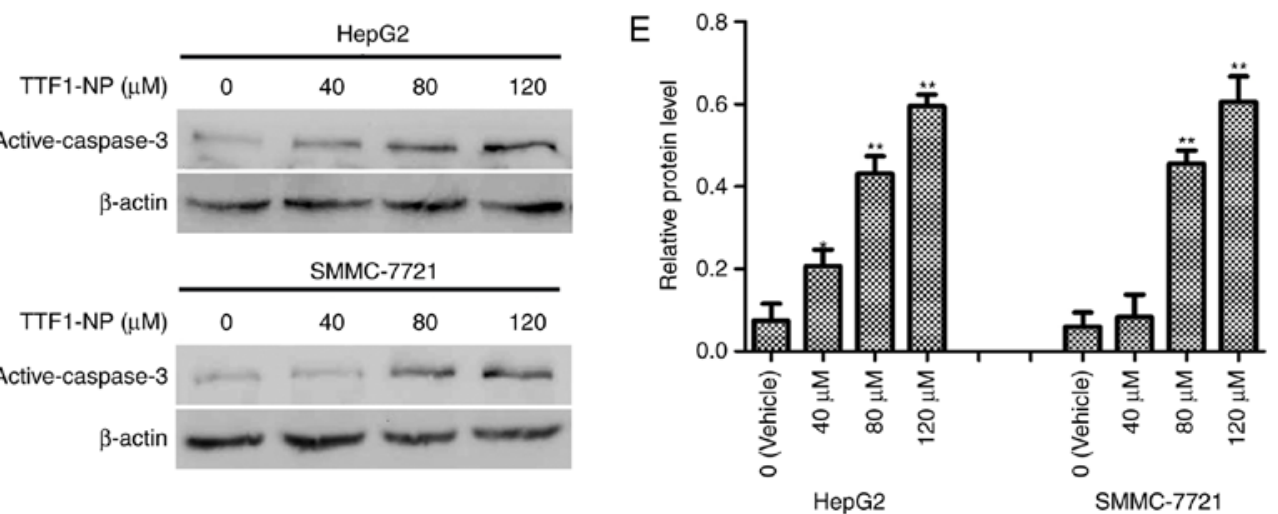

Figure 1. TTF1-NP inhibits cell viability and induces apoptosis in HepG2 and SMMC-7721 cells. (A) Chemical structure of TTF1-NP. (B) HepG2, SMMC-7721 were treated with $0,40,80$, and $120 \mu \mathrm{M}$ TTF1-NP for $24 \mathrm{~h}$, and cell viability was measured by MTT assay. ${ }^{*} \mathrm{P}<0.05,{ }^{* *} \mathrm{P}<0.01 \mathrm{vs}$. Vehicle. (C) HepG2 and SMMC-7721 cells were stained with Hoechst and observed by fluorescence microscopy (magnification, x400). Arrows indicate apoptotic cells. (D) HepG2 and SMMC-7721 cells were treated with TTF1-NP $(0,40,80,120 \mu \mathrm{M})$ for $24 \mathrm{~h}$ and the expression of active-caspase-3 was detected by western blotting. (E) Densitometry analysis of data in (D). Active-caspase-3 levels relative to $\beta$-actin were determined. Data presented are means $\pm \mathrm{SD}$. ${ }^{*} \mathrm{P}<0.05$, ${ }^{* * *} \mathrm{P}<0.01$ vs. Vehicle.

in HepG2 and SMMC-7721 cells (Fig. 1C-E). These results indicate that TTF1-NP induced apoptosis in a dose-dependent manner in HepG2 and SMMC-7721 cells.

TTF1-NP induces autophagy in HepG2 and SMMC-7721 cell lines. To determine whether TTF1-NP induces autophagy in HepG2 and SMMC-7721 cells, monodansylcadaverine (MDC) staining was used to detect preliminary autophagosomes. We observed increasing levels of positive staining for autophagic puncta with increasing TTF1-NP concentration in both cell lines (Fig. 2A). For further monitoring of autophagosome formation, HepG2 and SMMC-7721 cells were examined by transmission electron microscopy (TEM) or transfected with green fluorescent protein (GFP)-light chain 3 (LC3) plasmid.
The results showed that TTF1-NP induced formation of autophagosomes with double-layered membranes, and that the average number of GFP-LC3 puncta per cell was increased in both cell lines, compared with vehicle-treated cells (Fig. 2B-D). Furthermore, western blot analysis of the autophagy-related proteins LC3B I/II, Beclin-1, and p62 showed that TTF1-NP increased the protein levels of LC3B-II and Beclin-1, but decreased p62 levels (Fig. 2E and F). Collectively, these results indicate that TTF1-NP induced autophagy in HepG2 and SMMC-7721 cells.

Autophagy inhibition enhanced TTF1-NP-induced apoptosis in HepG2 and SMMC-7721 cells. It is reported that autophagy had a paradoxical effect on death or survival 
A
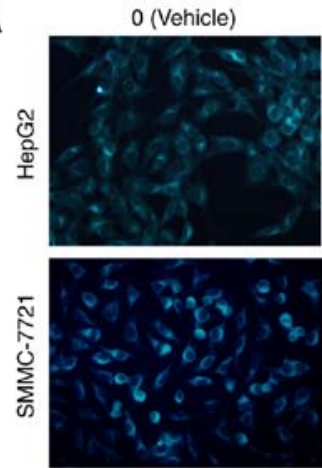

$40 \mu \mathrm{M}$
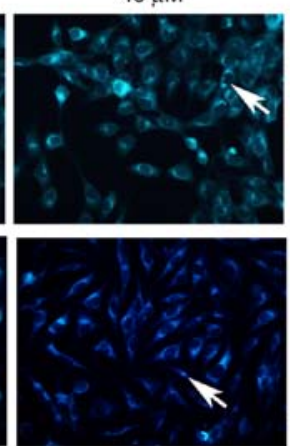

$80 \mu \mathrm{M}$
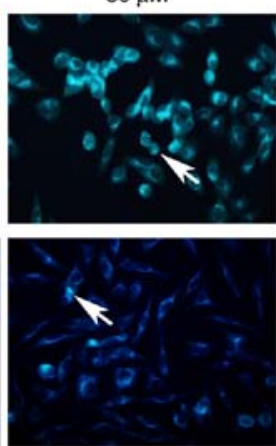

C
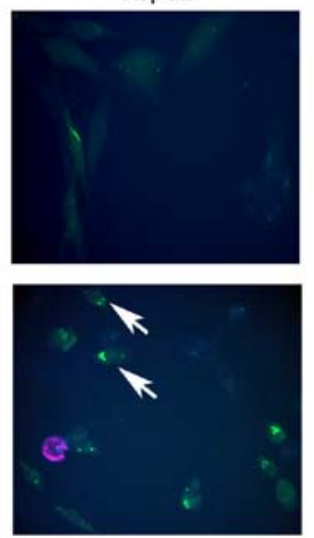

$120 \mu \mathrm{M}$
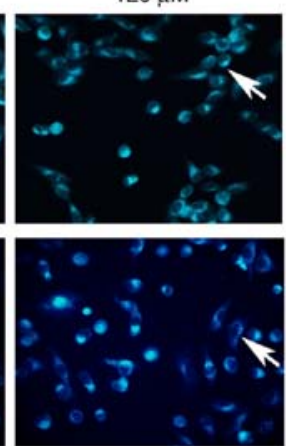

B
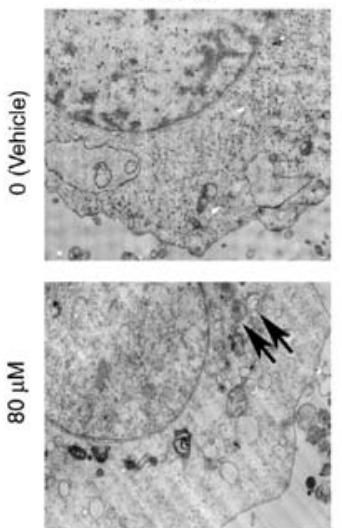

$(\times 2000)$
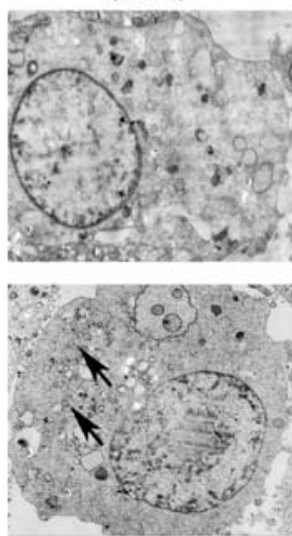

HepG2
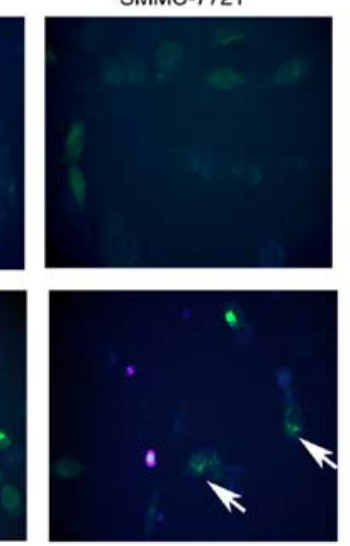

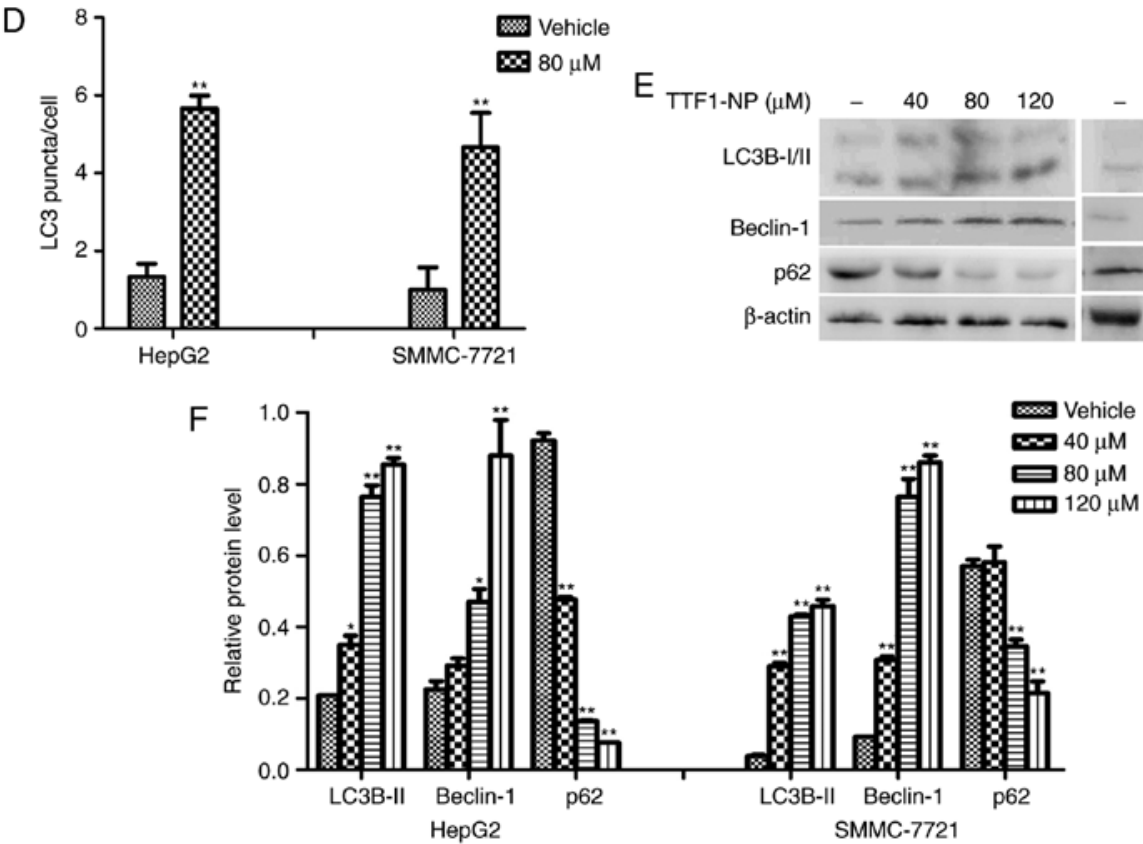

Figure 2. TTF1-NP induces autophagy in HepG2 and SMMC-7721 cells. (A) HepG2 and SMMC-7721 cells were treated with TTF1-NP (0, 40, 80, 120 $\mu$ M) for $24 \mathrm{~h}$, stained with MDC and observed by fluorescence microscopy (magnification, x400). (B) The formation of autophagosomes were observed in TTF1-NP-treated cells under transmission electron microscopy (TEM). (C) Cells were transfected with GFP-LC3 plasmid and treated with TTF1-NP for 24 h. GFP-LC3 puncta were observed by a fluorescence microscopy (magnification, $x 400)$. (D) The number of GFP-LC3 puncta was counted. Data represent the mean \pm SD. ${ }^{* *} \mathrm{P}<0.01$ vs. Vehicle. (E) HepG2 and SMMC-7721 cells were treated with TTF1-NP $(0,40,80,120 \mu \mathrm{M})$ for $24 \mathrm{~h}$ and the expression of LC3B I/II, Beclin-1, p62 was detected by western blotting. (F) Densitometry analysis of data in (E). LC3B-II, Beclin-1 and p62 levels relative to $\beta$-actin were determined. Data presented are means $\pm \mathrm{SD} .{ }^{*} \mathrm{P}<0.05,{ }^{* *} \mathrm{P}<0.01$ vs. Vehicle.

in cancer cells (8-10). To clarify the role of autophagy in TTF1-NP-induced apoptosis, we used the autophagy inhibitor 3-MA to prevent autophagy and co-treated with TTF1-NP for 24 h. Compared with TTF1-NP group, TTF1-NP co-treatment with 3-MA decreased cell viability (Fig. 3A). Furthermore, the result of flow cytometry assay showed that inhibition of autophagy significantly increased apoptotic cells in TTF1-NP+3-MA group comparing with TTF1-NP treatment 

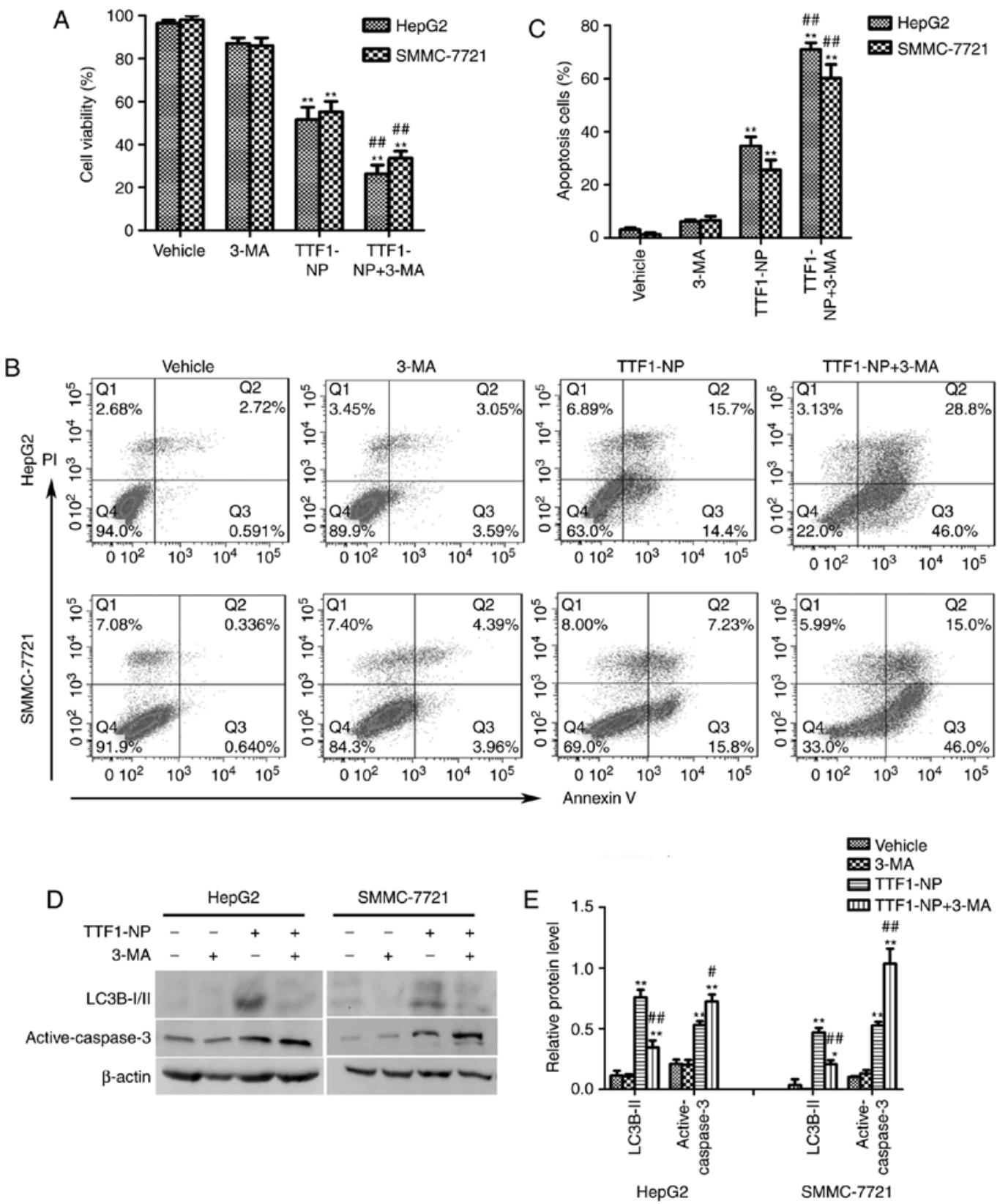

Figure 3. Autophagy inhibition enhances TTF1-NP-induced apoptosis in HepG2 and SMMC-7721 cells. (A) HepG2 and SMMC-7721 cells were pre-treated with $1 \mathrm{mM} 3$-MA for $1 \mathrm{~h}$ and treated with TTF1-NP $(80 \mu \mathrm{M})$ for $24 \mathrm{~h}$. Cells viability was measured by MTT assay. ${ }^{* *} \mathrm{P}<0.01 \mathrm{vs}$. vehicle; ${ }^{\# \#} \mathrm{P}<0.01 \mathrm{vs}$. TTF1-NP group. (B) The apoptotic cells were assessed by flow cytometry with Annexin V/PI staining. (C) Quantitative analysis of apoptosis in flow cytometry. Results are shown as mean $\pm \mathrm{SD} .{ }^{*} \mathrm{P}<0.05,{ }^{* *} \mathrm{P}<0.01$ vs. Vehicle. ${ }^{\# \#} \mathrm{P}<0.01$ vs. TTF1-NP group. (D) Western blot analysis for the expression of LC3B I/II and active-caspase-3 was detected. (E) Densitometry analysis of data in (D). LC3B-II, active-caspase-3 levels relative to $\beta$-actin were determined. Data presented are means $\pm \mathrm{SD} .{ }^{*} \mathrm{P}<0.05,{ }^{* *} \mathrm{P}<0.01$ vs. Vehicle; ${ }^{*} \mathrm{P}<0.05,{ }^{\# \#} \mathrm{P}<0.01$ vs. TTF1-NP group.

group (Fig. 3B, C). Additionally, TTF1-NP-induced caspase-3 activation was enhanced by 3-MA (Fig. 3D and E). Taken together, these results suggested that autophagy could have a protective effect in TTF1-NP-induced apoptosis in HepG2 and SMMC-7721 cells, prevention of autophagy could enhance the anticancer effect of TTF1-NP in human liver cancer cells.

TTF1-NP-induced autophagy and apoptosis are related to the Akt/mTOR pathway in HepG2 and SMMC-7721 cell lines. The Akt/mTOR pathway plays an important role in cell proliferation, apoptosis, and autophagy. To determine whether TTF1-NP-induced apoptosis and autophagy were involved in this signaling pathway, we examined the expression of
Akt, phosphorylated (p)-Akt (Ser473), mTOR and p-mTOR (Ser2448). The results showed that the levels of the p-Akt (Ser473) and p-mTOR (Ser2448) decreased in a dose-dependent manner in HepG2 and SMMC-7721 cells (Fig. 4A-C). Moreover, we used insulin to activate the $\mathrm{Akt} / \mathrm{mTOR}$ pathway and investigated the relationship between this pathway and autophagy or apoptosis. As shown in Fig. 4D-F, insulin significantly increased levels of p-Akt (Ser473) and p-mTOR (Ser2448) in both cell lines $(\mathrm{P}<0.05)$. When cells were pre-treated with TTF1-NP and then stimulated with insulin, the phosphorylation of Akt and mTOR increased compared with cells treated with TTF1-NP only, while levels of LC3B-II and active caspase-3 decreased in response to 
A
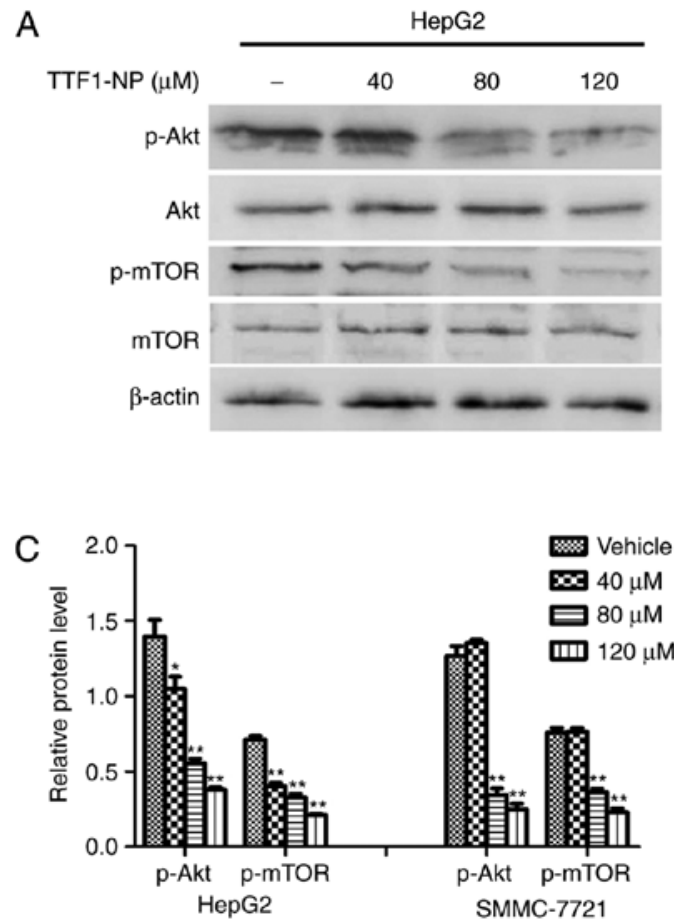

E

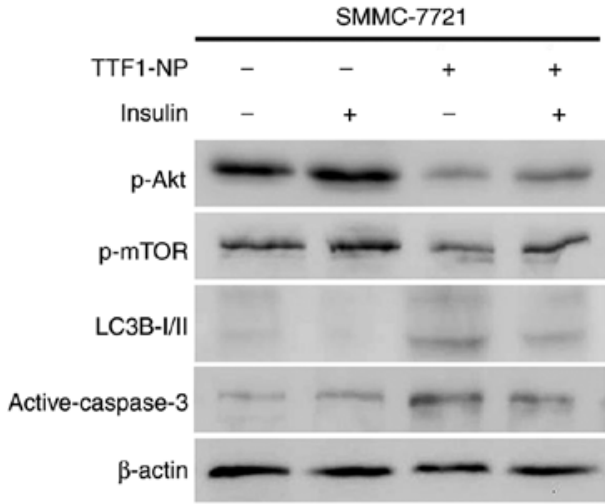

B

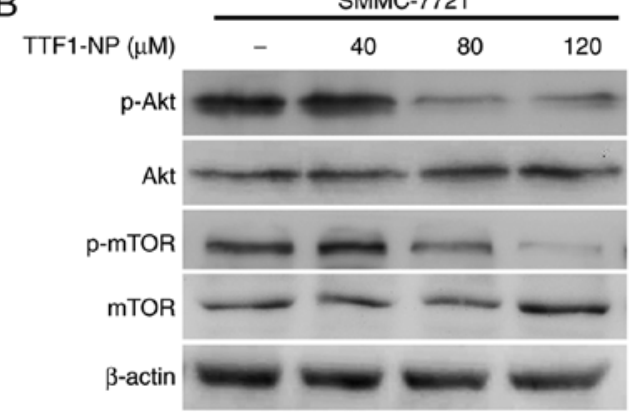

D

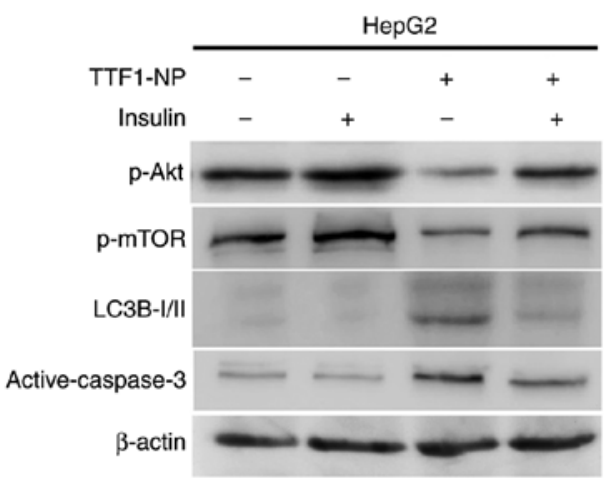

F

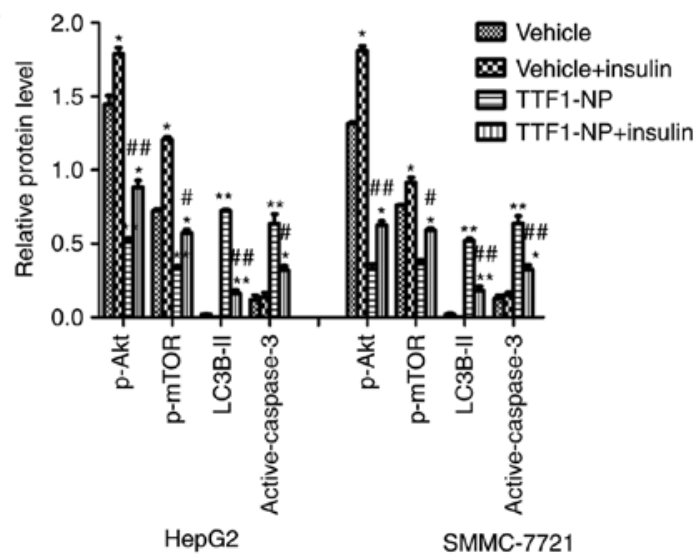

Figure 4. AKT/mTOR pathway is involved in TTF1-NP-induced autophagy and apoptosis in HepG2 and SMMC-7721 cells. (A and B) HepG2 and SMMC-7721 cells were treated with TTF1-NP $(0,40,80,120 \mu \mathrm{M})$ for $24 \mathrm{~h}$. Western blot analysis for the expression of Akt, p-Akt, mTOR and p-mTOR was detected. (C) Densitometry analysis of data in (A, B), p-Akt and p-mTOR levels relative to $\beta$-actin was performed. ${ }^{*} \mathrm{P}<0.05,{ }^{* *} \mathrm{P}<0.01$ vs. Vehicle. (D and E) HepG2 and SMMC-7721 cells were treated with $80 \mu \mathrm{M}$ of TTF1-NP or insulin, western blot analysis for the expression of p-Akt, p-mTOR, LC3B I/II and active-caspase-3 were detected. (F) Densitometry analysis of data in (D and E), p-Akt, p-mTOR, LC3B-II and active-caspase-3 levels relative to $\beta$-actin was performed. ${ }^{*} \mathrm{P}<0.05$, ${ }^{* *} \mathrm{P}<0.01$ vs. Vehicle; ${ }^{\#} \mathrm{P}<0.05,{ }^{\# \#} \mathrm{P}<0.01$ vs. TTF1-NP group.

insulin (Fig. 4C, D and F). Together, these results showed that the TTF1-NP-induced autophagy and apoptosis in HepG2 and SMMC-7721 cells may be mediated by the Akt/mTOR pathway.

TTF1-NP induces autophagy and apoptosis regulated by $J N K$ and ERK1/2. Many studies have reported that the MAPK pathway influences apoptosis and autophagy $(15,16)$. To examine the effect of TTF1-NP on the MAPK pathway, we evaluated the phosphorylation of JNK, ERK1/2 and p38 MAPK by western blotting. Levels of p-JNK (Thr183/Tyr185) increased and levels of p-ERK1/2 (Thr202/Tyr204) decreased in a concentration-dependent manner in response to TTF1-NP treatment (Fig. 5A and B). However, the level of p-p38 MAPK
(Thr180/Tyr182) did not change (Fig. 5A and B). To further investigate whether TTF1-NP-induced autophagy and apoptosis relied on JNK and ERK1/2, we pretreated cells with SP600125 and U0126 (a JNK and an ERK1/2 inhibitor, respectively) for $4 \mathrm{~h}$ followed by treatment with TTF1-NP for $24 \mathrm{~h}$. The results showed that levels of LC3B-II and active caspase-3 decreased in the TTF1-NP- and SP600125-treated cells, compared with cells treated with TTF1-NP only. However, U0126 enhanced active caspase-3, but had no significant effect on TTF1-NP-induced LC3B-II expression (Fig. 5C-F). Taken together, these results suggest that JNK was associated with the effect of TTF1-NP on autophagy and apoptosis, while ERK1/2 may be related to TTF1-NP-induced apoptosis in HepG2 and SMMC-7721 cells. 


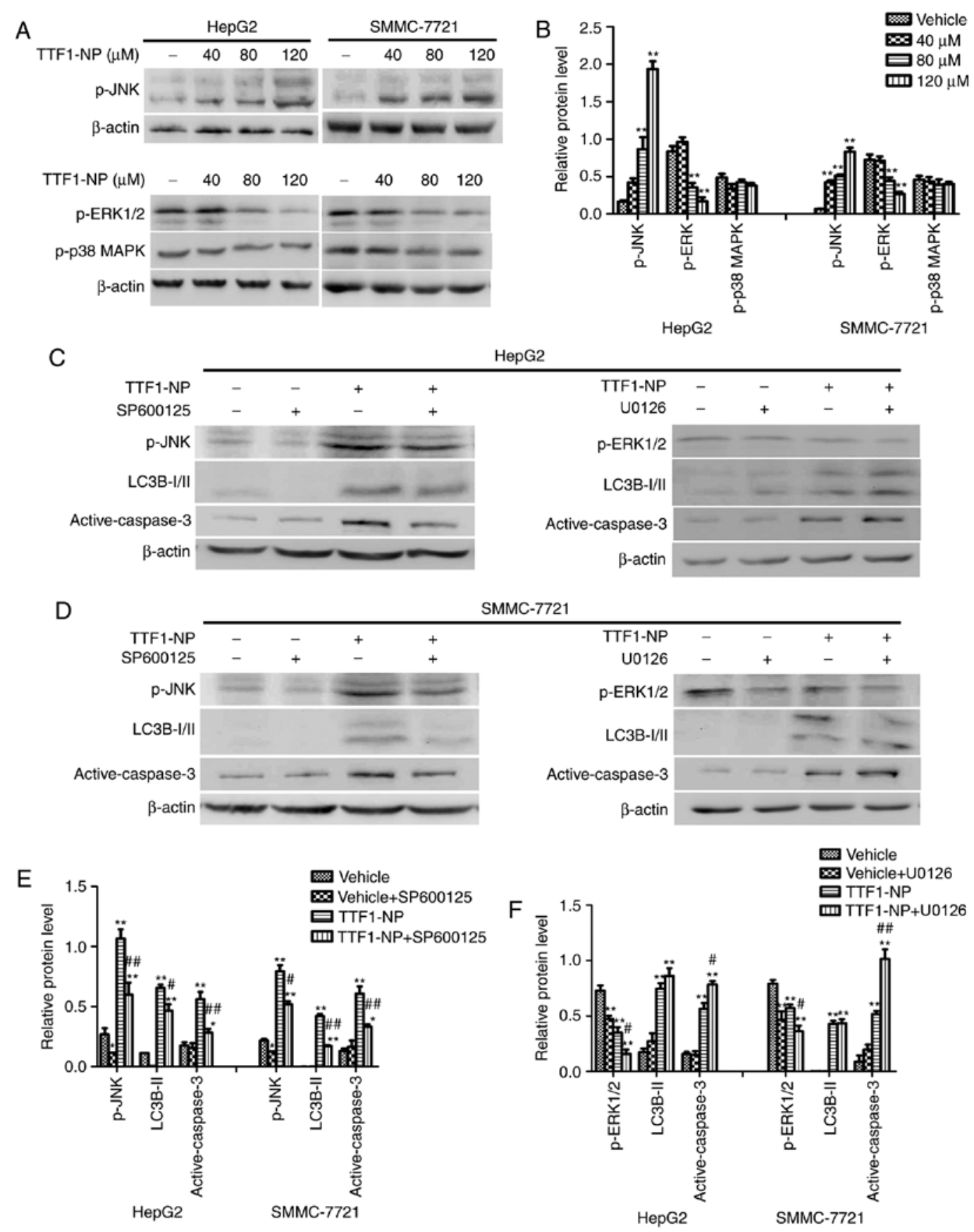

Figure 5. The JNK and ERK1/2 are involved in TTF1-NP-induced autophagy and apoptosis in HepG2 and SMMC-7721 cells. (A) HepG2 and SMMC-7721 cells were treated with TTF1-NP $(0,40,80,120 \mu \mathrm{M})$ for $24 \mathrm{~h}$. Western blot analysis for the expression of p-JNK, p-ERK and p-p38 MAPK were detected. (B) Densitometry analysis of p-JNK, p-ERK and p-p38 MAPK were performed. "P<0.05, ${ }^{* *} \mathrm{P}<0.01$ vs. Vehicle. (C) HepG2 were pre-treated with SP600125, U0126 or treated with $80 \mu \mathrm{M}$ of TTF1-NP, western blot analysis for the expression of p-JNK, p-ERK, LC3B I/II and active-caspase-3 were detected (D) SMMC-7721 were pre-treated with SP600125, U0126 or treated with $80 \mu \mathrm{M}$ of TTF1-NP, western blot analysis for the expression of p-JNK, p-ERK, LC3B I/II and active-caspase-3 were detected. (E and F) Densitometry analysis of data in (C and D) were performed in HepG2 and SMMC-7721 cells. "P<0.05, ${ }^{* *} \mathrm{P}<0.01$ vs. Vehicle; ${ }^{\#} \mathrm{P}<0.05,{ }^{\# \#} \mathrm{P}<0.01$ vs. TTF1-NP group.

\section{Discussion}

Hepatocellular carcinoma is the second leading cause of cancer-related mortality worldwide (17). Traditional postoperative chemotherapy is the current standard clinical treatment strategy. However, many drugs show limited efficacy, so there is an urgent need to develop more effective and lower-toxicity drugs or treatment approaches. We previously reported that TTF1-NP inhibited HepG2 cell proliferation by inducing apoptosis and inhibiting angiogenesis, migration and invasion in vitro and in vivo $(4,5)$. However, whether TTF1-NP induces autophagy in human hepatoma and the relationship between autophagy and apoptosis remain unknown.

Autophagy and apoptosis are topics of considerable interest in tumor research (18-20). In our study, TTF1-NP induced apoptosis, including changes to cell morphology and activation of the apoptosis-related protein caspase-3. Moreover, TTF1-NP induced autophagy. The number of puncta staining positive with MDC increased with increasing concentrations of TTF1-NP. However, MDC is not a specific 
dye for autophagosomes. TEM, the standard method for detecting autophagy $(21,22)$, is a more specific approach to examining autophagy. Our TEM results clearly show that autophagosomes with double-layered membranes appeared after TTF1-NP treatment. Furthermore, our data revealed that TTF1-NP increased the number of GFP-LC3B puncta in HepG2 and SMMC-7721 cells. Autophagy-related proteins such as LC3, Beclin-1 and p62 are important in the autophagy process. LC3 is homologous with Atg8 in yeast and is conjugated to phosphatidyl ethanolamine to form LC3-II, which is located in the autophagosome double membrane and positively correlated with autophagosome formation (23-25). Beclin-1 (Atg6) binds with class III PI3K as a complex to help form autophagosome structures (26). As another monitor of autophagic flux, p62 binds directly to LC3, and they are degraded during autophagy (27). Our study showed that TTF1-NP upregulated LC3B-II and Beclin-1 levels and decreased expression of p62. Collectively, these results suggest that TTF1-NP induced autophagy in both HepG2 and SMMC-7721 cells.

To further investigate the dual role of TTF1-NP-induced autophagy, 3-MA (an autophagy inhibitor) was used to prevent autophagy in both HepG2 and SMMC-7721 cells. Our results show that inhibiting autophagy increased the proportion of apoptotic cells and active caspase- 3 expression, which suggests that TTF1-NP-induced autophagy may play a protective role in TTF1-NP-induced apoptosis. Therefore, inhibition of autophagy may improve the efficacy of TTF1-NP treatment in human liver cancer cells.

The Akt/mTOR pathway plays a critical role in tumorigenesis. High expression of Akt frequently occurs in various tumors including hepatoma (13). mTOR is activated by Akt and negatively regulates autophagy (28). Extensive research has indicated that inhibiting the Akt/mTOR pathway contributes to autophagy in cancer cells $(29,30)$. In our study, TTF1-NP inhibited the Akt/mTOR pathway and decreased p-Akt (Ser473) and p-mTOR (Ser2448) levels in a dose-dependent manner. Furthermore, we used insulin to verify the influence of Akt/mTOR pathway on apoptosis and autophagy. As an activator, insulin binds with the insulin receptor and promotes PI3K conformational interconversion, which ultimately activates the Akt/mTOR pathway (31). Insulin significantly suppressed TTF1-NP-induced expression of LC3B-II and active caspase -3 , suggesting that the Akt/mTOR pathway may play an important role in TTF1-NP-induced autophagy and apoptosis in HepG2 and SMMC-7721 cells.

Many studies have shown that the MAPK pathway is involved in cell proliferation, apoptosis and autophagy $(32,33)$. Our experiments showed that TTF1-NP increased expression of p-JNK (Thr183/Tyr185) and decreased p-ERK1/2 (Thr202/Tyr204) in a concentration-dependent manner, but did not change p-p38 MAPK (Thr180/Tyr182) levels. Furthermore, TTF1-NP co-treatment with the JNK inhibitor SP600125 decreased caspase-3 activation, whereas co-treatment with the ERK inhibitor U0126 increased caspase-3 activation. Moreover, JNK inhibition reduced LC3-II levels, whereas U0126 did not affect TTF1-NP-induced LC3-II expression. The results suggest that effects on JNK may account for TTF1-NP-induced autophagy and apoptosis and ERK1/2 may account mainly for apoptosis in HepG2 and SMMC-7721 cells.
In conclusion, our study showed for the first time that TTF1-NP induced autophagy, and revealed the relationship between autophagy and apoptosis. Our results show that TTF1-NP induced a protective, apoptosis-related autophagy by regulating the Akt/mTOR pathway and JNK in HepG2 and SMMC-7721 cells. Co-treatment with an autophagy inhibitor reduced the effective treatment concentration of TTF1-NP. Thus, autophagy may be a potential therapeutic target of TTF1-NP in human hepatoma.

\section{Acknowledgements}

This study was supported by the NSFC (National Natural Science Foundation of China (code: 81460617).

\section{References}

1. Li Z, Cui FD and Zhang XW: Preparation technology of Sorbaria sorbifolia solid lipid nanoparticles. Lishizhen Med Materia Med Res 23: 2549-2550, 2012 (In Chinese).

2. Liu C, Li XW, Cui LM, Li LC, Chen LY and Zhang XW: Inhibition of tumor angiogenesis by TTF1 from extract of herbal medicine. World J Gastroenterol 17: 4875-4882, 2011.

3. Li Y, Bian L, Cui F, Li L and Zhang X: TTF1-induced apoptosis of HepG-2 cells through a mitochondrial pathway. Oncol Reps 26: 651-657, 2011.

4. Xiao B, Lin D, Zhang X, Zhang M and Zhang X: TTF1, in the form of nanoparticles, inhibits angiogenesis, cell migration and cell invasion in vitro and in vivo in human hepatoma through STAT3 regulation. Molecules 21: pii:E1507, 2016.

5. Xiao B, Liu C, Liu BT,Zhang X,Liu RR and Zhang XW: TTF1-NPs induce ERS-mediated apoptosis and inhibit human hepatoma cell growth in vitro and in vivo. Oncol Res 23: 311-320, 2016.

6. Yue H, Li W, Liu P, Gao J, Miao J and Zhao J: Inhibition of autophagy promoted sphingosylphosphorylcholine induced cell death in non-small cell lung cancer cells. Biochem Biophys Res Commun 453: 502-507, 2014

7. Yuan HX, Russell RC and Guan KL: Regulation of PIK3C3/VPS34 complexes by MTOR in nutrient stress-induced autophagy. Autophagy 9: 1983-1995, 2013.

8. Kim KY, Park KI, Kim SH, Yu SN, Park SG, Kim YW, Seo YK, Ma JY and Ahn SC: Inhibition of autophagy promotes salinomycin-induced apoptosis via reactive oxygen species-mediated PI3K/AKT/mTOR and ERK/p38 MAPK-dependent signaling in human prostate cancer cells. Int J Mol Sci 18: pii:E1088, 2017.

9. Zhang L, Wang H, Zhu J, Xu J and Ding K: Mollugin induces tumor cell apoptosis and autophagy via the $\mathrm{PI} 3 \mathrm{~K} / \mathrm{AKT} / \mathrm{mTOR} / \mathrm{p} 70 \mathrm{~S} 6 \mathrm{~K}$ and ERK signaling pathways. Biochem Biophys Res Commun 450: 247-254, 2014.

10. Ko A, Kanehisa A, Martins I, Senovilla L, Chargari C, Dugue D, Mariño G, Kepp O, Michaud M, Perfettini JL, et al: Autophagy inhibition radiosensitizes in vitro, yet reduces radioresponses in vivo due to deficient immunogenic signaling. Cell Death Differ 21: 92-99, 2014.

11. Liao G, Gao B, Gao Y, Yang X, Cheng X and Ou Y: Phycocyanin inhibits tumorigenic potential of pancreatic cancer cells: Role of apoptosis and autophagy. Sci Rep 6: 34564, 2016.

12. Liu Z, Wang F, Zhou ZW, Xia HC, Wang XY, Yang YX, He ZX, Sun T and Zhou SF: Alisertib induces G2/M arrest, apoptosis, and autophagy via PI3K/Akt/mTOR- and p38 MAPK-mediated pathways in human glioblastoma cells. Am J Transl Res 3: 845-873, 2017.

13. Gong L, Di C, Xia X, Wang J, Chen G, Shi J, Chen P, Xu H and Zhang W: AKT/mTOR signaling pathway is involved in salvianolic acid B-induced autophagy and apoptosis in hepatocellular carcinoma cells. Int J Oncol 49: 2538-2548, 2016.

14. He JD, Wang Z, Li SP, Xu YJ, Yu Y, Ding YJ, Yu WL, Zhang RX, Zhang HM and Du HY: Vitexin suppresses autophagy to induce apoptosis in hepatocellular carcinoma via activation of the JNK signaling pathway. Oncotarget 7: 84520-84532, 2016.

15. Hsieh MJ, Lin CW, Chen MK, Chien SY, Lo YS, Chuang YC, Hsi YT, Lin CC, Chen JC and Yang SF: Inhibition of cathepsin $\mathrm{S}$ confers sensitivity to methyl protodioscin in oral cancer cells via activation of $\mathrm{p} 38$ MAPK/JNK signaling pathways. Sci Rep 7: 45039, 2017. 
16. Hsieh MJ, Chien SY, Lin JT, Yang SF and Chen MK Polyphyllin $\mathrm{G}$ induces apoptosis and autophagy cell death in human oral cancer cells. Phytomedicine 13: 1545-1554, 2016.

17. Shen L, Zhang G, Lou Z, Xu G and Zhang G: Cryptotanshinone enhances the effect of Arsenic trioxide in treating liver cancer cell by inducing apoptosis through downregulating phosphorylated-STAT3 in vitro and in vivo. BMC Complement Altern Med 17: 106, 2017

18. Liu S, Fei W, Shi Q, Li Q, Kuang Y, Wang C, He C and Hu X: CHAC2, downregulated in gastric and colorectal cancers, acted as a tumor suppressor inducing apoptosis and autophagy through unfolded protein response. Cell Death Dis 8: e3009, 2017.

19. Tan J, Jiang X, Yin G, He L, Liu J, Long Z, Jiang Z and Yao K Anacardic acid induces cell apoptosis of prostatic cancer through autophagy by ER stress/DAPK3/Akt signaling pathway. Oncol Rep 38: 1373-1382, 2017.

20. Wang J, Tan X, Yang Q, Zeng X, Zhou Y, Luo W, Lin X, Song L, Cai J, Wang $\mathrm{T}$ and Wu X: Inhibition of autophagy promotes apoptosis and enhances anticancer efficacy of adriamycin via augmented ROS generation in prostate cancer cells. Int J Biochem Cell Biol 77: 80-90, 2016.

21. Wang KF, Yang H, Jiang WQ, Li S and Cai YC: Puquitinib mesylate (XC-302) induces autophagy via inhibiting the $\mathrm{PI} 3 \mathrm{~K} / \mathrm{AKT} / \mathrm{mTOR}$ signaling pathway in nasopharyngeal cancer cells. Int J Mol Med 36: 1556-1562, 2015.

22. Zhao R, Chen M, Jiang Z, Zhao F, Xi B, Zhang X, Fu H and Zhou K: Platycodin-D induced autophagy in non-small cell lung cancer cells via PI3K/Akt/mTOR and MAPK signaling pathways. J Cancer 6: 623-631, 2015.

23. Hsieh MJ, Chen MK, Chen CJ, Hsieh MC, Lo YS, Chuang YC Chiou HL and Yang SF: Glabridin induces apoptosis and autophagy through JNK1/2 pathway in human hepatoma cells. Phytomedicine 23: 359-366, 2016.

24. Kim YJ, Kang KS, Choi KC and Ko H: Cardamonin induces autophagy and an antiproliferative effect through JNK activation in human colorectal carcinoma HCT116 cells. Bioorg Med Chem Lett 25: 2559-2564, 2015.

25. Mizushima N and Yoshimori T: How to interpret LC3 immunoblotting. Autophagy 3: 542-545, 2007.
26. Mastorci K, Montico B, Faè DA, Sigalotti L, Ponzoni M, Inghirami G, Dolcetti R and Dal Co J: Phospholipid scramblase 1 as a critical node at the crossroad between autophagy and apoptosis in mantle cell lymphoma. Oncotarget 7: 41913-41928, 2016.

27. Reis FS, Lima RT, Morales P, Ferreira IC and Vasconcelos MH: Methanolic extract of ganoderma lucidum induces autophagy of AGS Human gastric tumor cells. Molecules 20: 17872-17882, 2015.

28. Ge D, Han L, Huang S, Peng N, Wang P, Jiang Z, Zhao J, Su L, Zhang S, Zhang Y, et al: Identification of a novel MTOR activator and discovery of a competing endogenous RNA regulating autophagy in vascular endothelial cells. Autophagy 10: 957-971, 2014.

29. Zhao D, Wang W, Wang H, Peng H, Liu X, Guo W, Su G and Zhao Z: PKD knockdown inhibits pressure overload-induced cardiac hypertrophy by promoting autophagy via AKT/mTOR pathway. Int J Biol Sci 13: 276-285, 2017.

30. Qin Y, Meng L, Fu Y, Quan Z, Ma M, Weng M, Zhang Z, Gao C, Shi X and Han K: SNORA74B gene silencing inhibits gallbladder cancer cells by inducing PHLPP and suppressing Akt/mTOR signaling. Oncotarget 8: 19980-19996, 2017.

31. Yao $H$, Han $X$ and Han $X$ : The cardioprotection of the insulin-mediated PI3K/Akt/mTOR signaling pathway. Am J Cardiovasc Drugs 14: 433-442, 2014.

32. Liu J, Chang F, Li F, Fu H, Wang J, Zhang S, Zhao J and Yin D: Palmitate promotes autophagy and apoptosis through ROS-dependent JNK and p38 MAPK. Biochem Biophys Res Commun 463: 262-267, 2015.

33. Zhang C, Jia X, Wang K, Bao J, Li P, Chen M, Wan JB, Su H, Mei Z and He C: Polyphyllin VII induces an autophagic cell death by activation of the JNK pathway and inhibition of PI3K/AKT/mTOR pathway in HepG2 cells. PLoS One 11: e0147405, 2016. 